\title{
A timely arrival for genomic medicine
}

\author{
Alan N. Mayer, MD, PhD, ${ }^{1,2}$ David P. Dimmock, MD, ${ }^{1,3,7}$ Marjorie J. Arca, MD, ${ }^{6}$ \\ David P. Bick, MD, ${ }^{1,3,7}$ James W. Verbsky, MD, PhD, ${ }^{1,4}$ Elizabeth A. Worthey, PhD, ${ }^{1,7}$ \\ Howard J. Jacob, PhD, ${ }^{1,7,8}$ and David A. Margolis, MD ${ }^{1,5}$
}

ln n this issue of $G I M,{ }^{1}$ we describe a young boy who presented with unusually aggressive inflammatory bowel disease refractory to medical and surgical treatment. To reach a diagnosis, we were compelled to use genomic technology that, at the time, was not a clinically validated test. This case stimulated many discussions within our group and institution on the boundary between research and clinical care. Many of the same issues were raised again during review of the manuscript, further shaping our thinking about how this case speaks to the broader issue of genomics in medical practice. The purpose of this commentary is to expound on these issues, hopefully to stimulate discussion within the wider medical community.

\section{CLINICAL COURSE AND THE DECISION TO SEQUENCE}

Our article ${ }^{1}$ provides an abbreviated history of this patient's unusual clinical course. In this limited space, it is difficult to convey the profound disability and suffering the child endured through numerous long hospital stays, and the resulting frustration that we experienced as we struggled to control the disease. Over a 3-year period, there were more than 100 surgical procedures, clinical consultations with physicians from around the world, innumerable informal discussions, weekly clinical care meetings, and informal e-mail consultations with world-leading experts. Despite these measures, we enjoyed little strategic success.

Allogeneic hematopoietic progenitor cell transplant was regularly brought up as a potential therapy, but two main barriers prevented us from moving forward. First, for the majority of the clinical course, the child was judged to be too ill to have a reasonable chance of surviving the first 100 days of the transplant process. Second, we lacked a firm diagnosis; hence, we could not predict whether bone marrow transplant would be likely to help. However, the risks of morbidity and mortality were high. Although the disease could be intermittently brought into remission, it was felt that eventually the child would succumb to drug toxicity, total parenteral nutrition liver disease, or recurrence. Thus, from a therapeutic standpoint, we were left with no viable long-term options.

The disease shared some similarities with Crohn disease, but the severity and tempo of disease progression was highly atypical.

From the Departments of ${ }^{1}$ Pediatrics, Sections of ${ }^{2}$ Gastroenterology, ${ }^{3}$ Genetics, ${ }^{4}$ Rheumatology, Allergy and Immunology, ${ }^{5} \mathrm{Hematology}$, Oncology and Bone Marrow Transplant, ${ }^{6}$ Surgery, Division of Pediatric Surgery, The Medical College of Wisconsin and Children's Hospital of Wisconsin, Milwaukee, Wisconsin; and ${ }^{7}$ Human and Molecular Genetics Center, ${ }^{8}$ Department of Physiology, The Medical College of Wisconsin, Milwaukee, Wisconsin.

Howard J. Jacob, PhD, Director, Human and Molecular Genetics Center, 8701 Watertown Plank Road, Milwaukee, WI 53226. E-mail: Jacob@mcw.edu.

Alan N. Mayer is currently at Department of Pediatrics, Pediatric Gastroenterology Division, Rush University Medical Center, Chicago, IL.

Disclosure: The authors declare no conflict of interest.

Published online ahead of print December 16, 2010.

DOI: 10.1097/GIM.0b013e3182095089
Exhaustive efforts to reach a diagnosis revealed numerous abnormalities in this child's immune system, but none of these were pathognomonic for a specific disease. Similarly, conventional genetic testing of numerous candidate genes had failed to reach an answer. Therefore, we decided the next logical step was to sequence the patient's exome (all known exons within the patient's genome).

\section{FROM DATA TO DIAGNOSIS}

Initially, we formulated the following hypothesis: the disease was likely to be a single gene disorder with a recessive mode of inheritance. As we were looking for a recessive disease with a population frequency of $<1: 10,000$, we could exclude genetic variants found in more than $1 \%$ of the general population as being causal of the child's disease. Initial analysis was limited to a set of 2006 target genes to reduce the risk of discovering off target information. After it was clear that none of the candidate genes harbored a pathogenic mutation, we broadened the analysis to include all known genes, eventually leading to the identification of a mutation in the XIAP gene. Because XIAP deficiency was not previously known to cause a severe Crohn-like phenotype, we then confirmed the loss of XIAP protein function in the patient's cells.

Having diagnosed the patient with XIAP deficiency, we needed to reorient the clinical approach to address the attendant risks of hemophagocytic lymphohistiocytosis (HLH), regardless of its role in the etiology of the patients inflammatory bowel disease. $^{2}$ Accordingly, we evaluated for Epstein-Barr Virus infection (negative to date) and considered approaches to chemoprophylaxis. We reviewed the intestinal pathology and bone marrow specimens, performed a liver biopsy, and established that there was no evidence of active HLH. Nevertheless, the data regarding the natural history of XLP2 suggest that this child had a high probability of death due to HLH in the future, an outcome that could be prevented by hematopoietic stem cell transplant. Therefore, this was the singular basis for the decision to perform a transplant. Furthermore, the link between XIAP and a loss of NOD2 signaling, a pathway implicated in Crohn disease, gave us hope that a transplant could improve the gastrointestinal condition as well.

\section{OUTCOME}

As of this writing, more than 5 months posttransplant, the patient has stably engrafted and is no longer transfusion dependent. Lansky performance status is $100 \%$. There is no clinical evidence of GI disease, although we recognize that the disease could recur over the ensuing months to years. Accordingly, we will be monitoring the patient closely for the foreseeable future.

\section{ETHICAL CONSIDERATIONS}

At the time we proposed whole-exome sequencing, this was not a clinically validated test, and questions arose about whether this was research. The relevant Institutional Review Board 
agreed with the team that, because the primary purpose of sequencing was clinical management and not secondarily generalizable knowledge, this was not research.

One of the intrinsic concerns of exome sequencing is diagnosing a disease that was not the target of an initial evaluation. In this case, making this diagnosis not only offered hope for the child's long-term survival but also brought the prospect of $\mathrm{HLH}$, a new life-threatening disease. Granted that the newly discovered risk predisposition was intrinsic to the underlying pathology, it did raise the question of how one systematically counsels families before testing about "off target" results.

Our current process for obtaining consent for clinical wholegenome sequencing has evolved from our approach to whole genome copy number analysis. Having the entire exome and ultimately the entire genome surveyed and potential risks identified, this is a new standard with respect to scale, but we believe not with respect to patient and parental rights. Consequently, the process for consent, in our institution, typically takes about 6 hours over several conversations. We take considerable time to educate the parents about the test, what it can and cannot offer, timeframe for results, and the risks of "off target" or unexpected results.

After considerable discussions with ethicists at both of our internal institutions and external experts, the decision was made that parents are in the best position to make a decision about what "off target" test results will be made available to them. Consequently, before testing, a plan is established with the parents about what information is returned based on three broad categories.

1. Information that will directly impact the care of the child in childhood (such as XIAP) is always returned.

2. Information on adult onset disease that would be actionable in adulthood, which may have significant implications for the parents' own current health maintenance may be returned electively. An example would be pathogenic mutations in $B R C A 1$, which predisposes carriers to breast cancer but can be prevented by elective mastectomy.

3. Mutations in adult onset genes for which there is not currently a preventative treatment can be returned electively. An example would be pathogenic mutations in PARK1, which predisposes to Parkinson disease.

We schedule at minimum annual follow-up in our genetics clinic for all families in which whole-genome sequencing has been performed and discuss further options for information disclosure at these meetings, in addition to providing options to the child when they reach the age of majority. We think this strategy is sound for the present, but further research is warranted into the risks and benefits of such strategies as it becomes possible to interpret more variants found in the genome.

\section{$\cos T$}

It is always difficult to perform a cost/benefit analysis for an individual case. When compared with most single laboratory tests, the cost of exome or whole-genome sequencing is high. But the cost of sequencing continues to plummet, such that the current cost of exome or whole-genome sequencing in a clinical laboratory is significantly less than the battery of clinical DNA tests sent on this child. Furthermore, the data sets used to interpret individual sequence variants will expand. Although this example is retrospective, we expect that as costs for wholegenome sequencing and subsequent analysis fall, there will be a stronger cost argument for whole-genome sequencing as the initial diagnostic DNA test. Indeed, for a clinical diagnosis that presents significant locus heterogeneity (i.e., immune deficiency), whole-genome sequencing may be more cost effective than iteratively testing numerous candidate genes.

\section{CONCLUSIONS}

This case began with a disease and ended with a molecular diagnosis. The tools available to make this diagnosis have evolved so rapidly that they were not available when the child first presented 4 years ago. Although the history and physical examination remain a cornerstone of diagnosis, we are now entering an era in which comprehensive genomic analysis at the nucleotide level, such as whole-exome sequencing, has been added to the clinician's armamentarium. Our view from the bedside was that exome sequencing enabled us to offer a treatment based on a diagnosis rooted in disease natural history and laboratory science. To do so, we needed to marshal the talents of a large number of scientists, allied health professionals, and physicians. We learned a tremendous amount about sequencing for clinical utility that continues to ripple through our institution. Many of the lessons learned, such as the utility of pathway and literaturecompiled gene lists, management of parental anxiety, test result disclosure, and evaluation of utility can only be understood in the context of clinical practice. Accordingly, we are developing a new strategy as well as formal policies and procedures to guide our approach to future cases based on our experience with this case. We are confident that genomic sequencing will have a growing role in establishing the correct diagnosis for patients and, most importantly, improving outcomes.

\section{REFERENCES}

1. Worthey EA, Mayer AN, Syverson GD, et al. Making a definitive diagnosis: successful clinical application of whole exome sequencing in a child with intractable inflammatory bowel disease. Genet Med 2011;13:255-262.

2. Rigaud S, Fondaneche MC, Lambert N, et al. XIAP deficiency in humans causes an X-linked lymphoproliferative syndrome Nature 2006;444:110-114. 